\title{
Factores que influyen en la demanda de resultados de citologías cervicouterinas en las IPS de Villavicencio'
}

\author{
Mauricio Cavieles² \\ Sydney Gutiérrez ${ }^{3}$ \\ Ana Castro 4
} de resultados de citologías cervicouterinas en las IPS de Villavicencio. Investig Enferm. Imagen Desarr. 2014;16(2):49-63. doi:10.11144/Javeriana.IE16-2.fidr

1. Artículo de investigación. Recibido: 16 de marzo de 2014. Revisado: 3 al 20 de abril de 2014. Aceptado: 22 de julio de 2014.

2. Enfermero. Especialista en Auditoría de Servicios de Salud. Enfermero ESE Solución Salud, Mesetas, Meta. Correo electrónico: macape321@hotmail.com.

3. Enfermera. Especialista en Auditoría de Servicios de Salud. Enfermera EPS Mallamas, Villavicencio, Colombia. Correo electrónico: alejita187@hotmail.es.

4. Enfermera. Magíster y doctora en Salud Pública. Catedrática de la Especialización en Seguridad y Salud en el Trabajo, Universidad de los Llanos, Villavicencio, Colombia. Correo electrónico: acastro@unillanos.edu.co. 


\section{Resumen}

Objetivo: Analizar los factores que influyen en la demanda de resultados de citologias cervicouterinas en las IPS de primer nivel de Villavicencio. Metodología: El diseño fue descriptivo-transversal, retroprospectivo, con enfoque cualicuantitativo, con una población de 1162 resultados no reclamados durante el 2008. Se obtuvo la muestra aleatoria de 322 resultados y sus correspondientes usuarias. Los instrumentos retomaron las variables contenidas en el formato de citología y en una encuesta con variables que podrían estar influyendo en la no reclamación, aplicada vía telefónica. Resultados: Se identificó que la mayoría de la población tenía condiciones sociales, económicas y educativas desfavorables y las IPS no cumplian con la entrega de los resultados en la fecha establecida. Conclusiones: Hay determinantes administrativos para resolver e intervenir durante la consulta haciendo hincapié en la importancia de los resultados para un seguimiento regular y, de ser necesario, un tratamiento oportuno.

Palabras clave: conocimiento de resultados; citología; tamizaje masivo

\section{Factors Influencing the Demand for Cervical Cytology Results in The Health Providing Institutions of Villavicencio}

\section{Abstract}

Objective: To analyze the factors influencing the demand for cervical cytology results in first-level IPS in Villavicencio. Methods: The design was cross-sectional descriptive, retro-prospective with quality-quantitative approach, with a population of 1162 unclaimed results during 2008. A random sample of 322 users and their corresponding results was obtained. Instruments retook the variables from the cytology form and a phone survey with variables that could be influencing the patients not claiming the results. Results: It was found that most of the population had adverse social, economic and educational conditions and IPS did not meet the delivery of results on the due date. Conclusions: There are administrative determinants to solve and intervene during the consultation by emphasizing the importance of the results for regular monitoring and, if necessary, early treatment.

Keywords: knowledge of results; cytology; mass screening 


\section{Fatores que influenciam na demanda de resultados de citologias da cérvice uterina nas IPS de Villavicencio}

\section{Resumo}

Objetivo: Analisar os fatores que influenciam na demanda de resultados de citologias de cérvice uterinas nas IPS de primeiro nível de Villavicencio, Colômbia. Metodologia: O desenho foi descritivo-transversal, retroprospetivo, com enfoque quali-quantitativo, com uma população de 1162 resultados não requestados durante o 2008. Obteve-se amostra aleatória de 322 resultados e suas correspondentes utentes. Os instrumentos retomaram as variáveis contidas no formato de citologia e em inquérito com variáveis que poderiam estar a influir no desinteresse por procurá-los, aplicado por contato telefónico. Resultados: Observou-se que a maioria da população tinha condições sociais, económicas e educativas desfavoráveis e as IPS (provedoras de serviços de saúde) não cumpriam com a entrega dos resultados na data estabelecida. Conclusões: Têm determinantes administrativas para resolver e intervir durante a consulta remarcando a importância dos resultados para um seguimento regular e, quando necessário, um tratamento oportuno.

Palauras chave: conhecimento de resultados; citologia; triagem em massa 


\section{Introducción}

Actualmente en el país el cáncer uterino continúa siendo una de las principales causas de muerte, a pesar de ser evitable y curable. Los esfuerzos por contrarrestarlo son grandes; sin embargo, se requieren programas accesibles y a bajo costo que detecten oportunamente lesiones precursoras, como la técnica de Papanicolaou en mujeres asintomáticas, y que por la misma línea se continúe con el tratamiento y seguimiento de la enfermedad. Esto es lo que se espera no solo en Colombia, sino en Latinoamérica (1). Dada su importancia en el país, se han realizado varios estudios de prevalencia, factores de riesgo y protectores en diferentes poblaciones $(2,3)$.

Este trabajo pretende contribuir al esclarecimiento de las razones por las cuales algunas mujeres que accedieron al examen de la citología, en alguna de las nueve instituciones prestadoras de servicios de salud (IPS) que hacen parte de la empresa social del Estado (ESE) municipal de Villavicencio, no reclamaron los resultados, lo que pudiera ser un factor en contra de la salud y contributivo de la situación actual de alta mortalidad por cáncer cervicouterino. Esto, a través del análisis de los factores que influyen en dicho trámite. La importancia de este trabajo radica en la posibilidad de encontrar los obstáculos existentes en la tercera fase (demanda de resultados) del proceso (examen de citología), cuyo conocimiento puede ser aplicable a la solución del problema.

El estudio descriptivo planteado ayuda, entre otros aspectos, a conocer la influencia de las características de la población objeto en las estrategias de prevención, sus implicaciones para el desarrollo de los programas de salud y proporciona información útil para investigadores e instituciones sobre cómo asegurar una respuesta positiva de la población al cuidado de su propia salud.

Son varios los estudios que mencionan aspectos relevantes sobre la estrategia educativa (4-8) y sobre la toma de la muestra $(9,10)$. Estos abordan temas que resaltan su importancia, evidencian defectos presentados o sugieren soluciones a dichos problemas. Por otro lado, en la literatura sobre "demanda de los resultados de citología" no se encontraron hallazgos, a no ser que se aborden de manera secundaria o se mencionen someramente. Esta situación representa un "vacío" en el conocimiento sobre la respuesta de la población objeto a los programas de tamizaje de cáncer de cuello uterino, vitales en la detección precoz de este tipo de cáncer, ya que si no se reclaman los resultados, se dificulta ofrecer una intervención oportuna.

\section{Materiales y métodos}

Se llevó a cabo un estudio descriptivo retroprospectivo transversal con el fin de analizar la información de las citologias (exámenes de tamizaje) que no habían sido reclamadas durante el 2008. A su vez, se contactó vía telefónica a las usuarias que correspondían a los exámenes sorteados, y el enfoque fue cualicuantitativo, esto es, se consideraron variables cualitativas y cuantitativas con sus correspondientes indicadores. La población hace 
referencia al conjunto de formatos de citología (N) de 1162 almacenados en las nueve IPS de la ESE municipal de Villavicencio.

El tamaño de la muestra ( $n$ ) se estableció mediante la función Statcalc del programa EpiInfo (versión 6.04), considerando los siguientes parámetros: una población (n) de 1162, un nivel de confianza (Z) del $95 \%$ y un margen de error (E) del $5 \%$, la cual arrojó un $n$ de 322. Se reemplazó el 30\% (97), que no respondió a la llamada telefónica o simplemente no quiso dar información, que se sustituyó haciendo llamadas de acuerdo con el intervalo determinado para la muestra aleatoria inicial. Así se mantuvo el $n$ de la muestra. Siempre se conservó la correspondencia de resultado no reclamado-usuaria que respondía la entrevista telefónica. Las unidades de análisis fueron los resultados de las citologías de cuello uterino no reclamados y las respectivas usuarias.

Se elaboró un formato digital a través del programa EpiInfo (versión 6.04), que emulaba el formulario institucional para la toma de citologia, en el que se consideraron todos los datos de identificación y localización de la usuaria. Con previa autorización de la ESE municipal, se asistió a cada IPS pública y se consignaron los datos de cada uno de los resultados no reclamados y obtenidos por sorteo; de esta forma se conformó una base de datos virtual, que se confrontó con el libro de registro, a fin de constatar la coherencia de los datos.

Posteriormente se elaboró una encuesta sencilla, para ser aplicada vía telefónica, que rescataba las características más importantes de la población en estudio: estado civil, edad, nivel económico y educativo, número de hijos, personas a cargo, jornada laboral (cuantitativas); las variables relacionadas con la no reclamación: cambio de residencia, accesibilidad a la IPS, información sobre la citología uterina, grado de importancia atribuido tanto a la toma de citología como a la demanda del resultado, concepto sobre el personal de salud, popularidad del cumplimiento en la entrega, concepto sobre la citología, razón atribuida a la demanda de los resultados, periodicidad de consulta (cualitativas). Se construyeron para las variables cualitativas indicadores acordes a su naturaleza, por ejemplo: las que incluian importancia atribuida a la toma de citología: mucha $(9,10)$; significativa (6 a 8); poca (1 a 5); ninguna (0); accesibilidad a la IPS: fácil o difícil, y así para las demás variables se construyeron indicadores. A la encuesta se le hizo una prueba piloto, en población similar, pero del primer bimestre de 2009, producto de lo cual se le realizaron ajustes.

Los datos obtenidos se consignaron en una "vista" del programa EpiInfo versión 6.04, que correspondia al instrumento aplicado. Mediante las funciones del programa se obtuvo el análisis univaridado y, posteriormente, se cruzaron las variables: importancia asignada a la toma de citología uterina y la importancia de recogerla. Los resultados se presentaron en tablas y figuras, en las cuales se evidencia el porcentaje de usuarias que respondieron cada uno de los items de los indicadores.

Los aspectos éticos contemplados fueron: consentimiento informado y confidencialidad, donde se les explicó el objetivo del estudio y el derecho 
que les asistía de aceptar o no participar en el estudio. Igualmente, que se tomaba la información con fines académico-investigativos y de contribuir con estrategias tendientes a mejorar el programa de tamizaje para cáncer cervicouterino.

\section{Resultados}

En la tabla 1 se presenta la distribución sociodemográfica de la población estudiada.

TABLA 1. Distribución porcentual de características sociodemográficas de la población estudiada

\begin{tabular}{|c|c|c|}
\hline Variable de estudio & \multicolumn{2}{|c|}{ Medidas de resumen } \\
\hline Edad mediana $\pm \mathrm{DE}$ & 35 & $\pm 9,5$ \\
\hline $16-20$ & 48 & 14,9 \\
\hline $21-25$ & 53 & 16,4 \\
\hline $26-30$ & 42 & 13,0 \\
\hline $31-35$ & 148 & 46,1 \\
\hline $35 y+$ & 31 & 9,6 \\
\hline \multicolumn{3}{|c|}{ Educación } \\
\hline Analfabeta & 7 & 2,1 \\
\hline Primaria incompleta & 77 & 23,9 \\
\hline Primaria & 40 & 12,3 \\
\hline Secundaria incompleta & 121 & 37,8 \\
\hline Secundaria completa & 54 & 16,8 \\
\hline Técnicas & 20 & 6,2 \\
\hline Universitaria & 3 & 0,9 \\
\hline \multicolumn{3}{|c|}{ Estado civil } \\
\hline Soltero & 66 & 20,4 \\
\hline Casado & 87 & 27,0 \\
\hline Viudo & 5 & 1,5 \\
\hline Separado & 16 & 4,9 \\
\hline Unión libre & 144 & 44,7 \\
\hline Divorciado & 4 & 1,2 \\
\hline \multicolumn{3}{|c|}{ Ingreso } \\
\hline No devengan & 176 & 54,7 \\
\hline$>1 \mathrm{SMLV}$ & 82 & 25,5 \\
\hline $1 \mathrm{SMLV}$ & 55 & 17,0 \\
\hline 2 SMLV & 9 & 2,8 \\
\hline
\end{tabular}

DE: desviación estándar; SMLV: salario mínimo legal vigente.

Fuente: Castro A, Cavieles M, Gutiérrez S. Factores que influyen en la demanda de los resultados de las citologías cervicouterinas en las IPS de Villavicencio durante el año 2008. Villavicencio: Programa de Enfermería, Universidad de los Llanos; 2009. 
Según los resultados referentes al estado civil de esta población, el $71,7 \%$ de las mujeres encuestadas cuenta en el momento con una pareja estable. Esta tendencia difiere de lo observado en la Encuesta Nacional de Demografía y Salud 2010 (11), donde solo el 51,5\% son casadas o viven en unión, en el grupo de mujeres en edad fértil (15-49 años).

En relación con las edades de las mujeres, la moda se ubicó en el intervalo de 31-35 años. Es importante notar que el grupo de 26-35 representa el $59,1 \%$, lo que lo convierte en el grupo predominante, jóvenes y en edad fértil.

En lo que respecta a nivel educativo alcanzado, se pudo observar un porcentaje de analfabetismo bajo, así como bajas tasas de cobertura en todos los niveles educativos, lo que implica un gran porcentaje de mujeres con estudios incompletos: secundaria incompleta $(37,8 \%)$ y primaria incompleta (21,4\%). Juntos reflejan un problema en común: deserción escolar.

La mayoría de la población no tiene una preparación académica adecuada que le brinde oportunidades frente a la vida; por otra parte, el nivel educativo influye de manera importante en sus actitudes y prácticas relacionadas con la salud (12).

En cuanto al ingreso económico, la mayoría de las mujeres encuestadas $(54,6 \%)$ refiere no tener ninguna remuneración económica, lo que implica alta dependencia, y de las que devengan (45,6\%), en su mayoría solamente obtienen ingresos que apenas aseguran las necesidades básicas de sobrevivencia. En concordancia con el Instituto Nacional de Cancerología, el cáncer de cuello uterino afecta principalmente a las mujeres de bajo nivel económico (13).

E1 90,6\% de las mujeres no cambiaron de residencia durante el último año; mientras que un $9,4 \%$ sí lo hizo. Esto significa que no es un factor muy influyente en la demanda de resultados. Sin embargo, las deficiencias en el seguimiento de las pacientes, en especial de aquellas con resultado anormal, son un alto riesgo a la salud. Por otro lado, no siempre las mujeres dejan consignados datos ciertos sobre cómo localizarla y tampoco avisan un cambio de domicilio o teléfono y el personal del centro asistencial no recoge de forma completa la información.

Si se tiene en cuenta que, de acuerdo con estudios internacionales $(8,14)$, la pérdida del seguimiento de una paciente con citología anormal indica que gran parte del programa ha fallado, deben revisarse y modificarse los métodos y prácticas en este campo.

Cuando se les preguntó a las mujeres sobre cómo consideraban el acceso a la IPS, el 51,3\% lo consideró fácil, y el 48,7\%, difícil. No se evidencia un predominio significativo de alguno de los dos indicadores, lo cual le confiere ambigüedad a la variable, y la invalida para producir un juicio discriminativo. Desde estos términos, su influencia ante la no reclamación de resultados estaría determinada por la presencia de otros factores.

En relación con la jornada laboral, el 56,8\% refiere no tener empleo, el $31 \%$ trabaja tiempo completo y el $12,1 \%$ trabaja medio tiempo. Se observó que la mayoría de las mujeres no tenía una ocupación formal; sin embargo, algunas mujeres no consideraban las tareas domésticas como una ocupa- 
ción, aun cuando estas terminan ejerciéndose como cualquier jornada laboral, de manera que habría que incluirlas implícitamente en el análisis. Solo la tercera parte de la población del estudio refirió tener una jornada laboral completa, lo que representa una clara limitante de tiempo. Seguramente, sin importar la actividad, las mujeres se ocupan en un considerable periodo, lo que hace este factor determinante en nuestra búsqueda.

Con respecto al número de hijos, el 26,7\% de las mujeres encuestadas refiere tener más de 3 hijos, seguido de las mujeres con 1 hijo (24,8\%), con 2 hijos (22,9\%) y mujeres con 3 hijos (17,7\%). E1 7,7\% refiere no tener hijos. Si bien la disponibilidad de tiempo disminuye en relación directa con el número de hijos, esto puede no ser obstáculo para reclamar los resultados, si existe una conciencia de salud en las usuarias.

Al considerar las personas a cargo: el $50 \%$ de las mujeres tiene 1-2 personas; el 29,5\%, 3-4 personas, y el 7,1\%, 5 o más personas. Solamente el $9,3 \%$ de las mujeres no tiene responsabilidades de este tipo. En un porcentaje mínimo de las mujeres no hay personas a cargo, dato que se acerca bastante al porcentaje de mujeres sin hijos. Estas dos variables se comportan de una manera similar, hasta el punto que puedan unificarse, validando los hijos dentro de las personas a cargo. No obstante, los dos, juntas o superpuestas, permiten valorar el grado de responsabilidad de las mujeres a estudio.

Respecto al conocimiento que tienen las mujeres acerca del examen de la citología: el 49,3\% afirmó tener suficiente, el 38,8\% mencionó saber poco sobre el tema y el $11,1 \%$ dijo no saber nada al respecto. El conocimiento, seguramente, les permite admitir la importancia del examen. Es un hecho que las estrategias educativas han permitido el acceso de dicho conocimiento a la población objeto, aunque no en su totalidad, y han colocado en desventaja a quienes no cuentan con la información, lo que implica un pequeño, pero significativo grupo al cual la estrategia no ha tocado por alguna razón y requiere intervención hasta lograr total cobertura con información preventiva.

E1 $77,6 \%$ de las mujeres refiere que la toma de la citología es muy importante; del mismo modo, el $78,8 \%$ atribuye un alto nivel de importancia a la demanda del resultado; el $15,5 \%$ da una importancia significativa a la toma del examen, y el $16,4 \%$ da la misma importancia a la demanda. La tendencia continúa con el indicador de poca importancia, con un $5,2 \%$ atribuido a la toma y un 3,4\% atribuido a la demanda. E1 1,5\% de las mujeres considera que la toma de la citología no tiene importancia, junto a un $0,9 \%$ que piensa lo mismo de la demanda del resultado. De estas variables relacionadas, lo más relevante es el alto porcentaje de usuarias que confiere gran importancia tanto a una como a otra (toma y demanda).

Cuando se les preguntó a las mujeres sobre lo que pensaban del personal de salud que las atendió en el examen de citología, el 81,9\% refirió haber sido tratado con amabilidad, el 17\% manifestó que el personal mantuvo el respeto, el 0,3\% dijo haber sido tratado con apatía y el 0,6\% se sintió irrespetada de algún modo. 


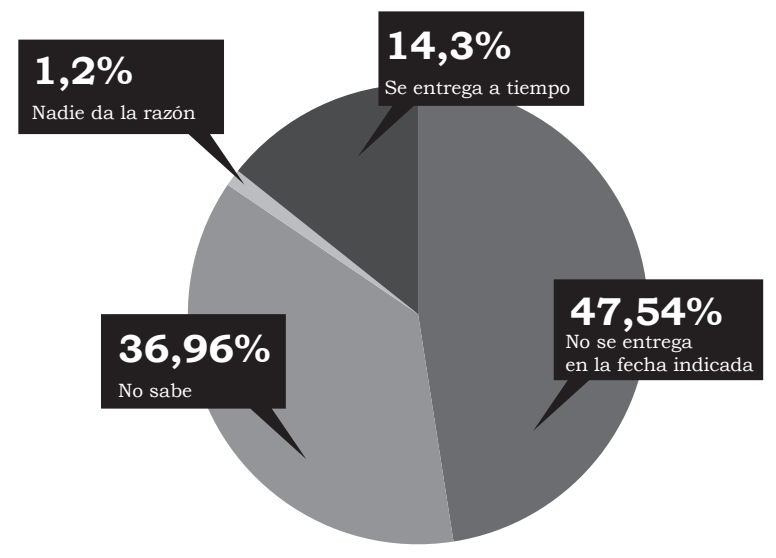

Figura 1. Distribución porcentual de citologías no reclamadas según popularidad del cumplimiento de la entrega de resultados de la población

Fuente: Castro A, Cavieles M, Gutiérrez S. Factores que influyen en la demanda de los resultados de las citologías cervicouterinas en las IPS de Villavicencio durante el año 2008. Villavicencio: Programa de Enfermería, Universidad de los Llanos; 2009.

En relación con la popularidad de la entrega de los resultados, el $47,5 \%$ dijo haber escuchado que los resultados no se entregaban en la fecha indicada, frente a un $14,2 \%$ de mujeres que escucharon que los resultados se entregaban a tiempo (figura 1). El concepto de las mujeres acerca de la entrega de resultados no favorece la credibilidad de estas instituciones, pues el incumplimiento impacta negativamente su popularidad.

En cuanto a la periodicidad con que las mujeres acuden a consulta médica, el 91,6\% de las encuestadas asistía al médico solamente cuando se enfermaban, el 6,2\% dijo acudir una vez al año y el 2,1\% iba cada mes. Se detecta una escasa inclinación al control médico periódico; se asiste según necesidad por aparición de síntomas, lo que no supone una cultura de la prevención. El comportamiento no refleja autocuidado de la salud, lo cual representa un riesgo para el caso de enfermedades que no se manifiestan tempranamente, como el cáncer.

El 58,6\% de las mujeres consideraba la citología un requisito solicitado; el $15,2 \%$ se refirió a la citología como necesaria, pero no se la realiza periódicamente; el 13,3\% la cree necesaria y se la realiza periódicamente, y el $12,7 \%$ la concibe como necesaria, se la realiza, pero no va por los resultados. En general, las mujeres consideran la citología un requisito solicitado, a pesar de que muchas de ellas conocen la importancia de la toma y la demanda del resultado. Al preguntarles a las mujeres sobre la razón por la cual no fueron por los resultados, las respuestas obtenidas se muestran en la figura 2. 


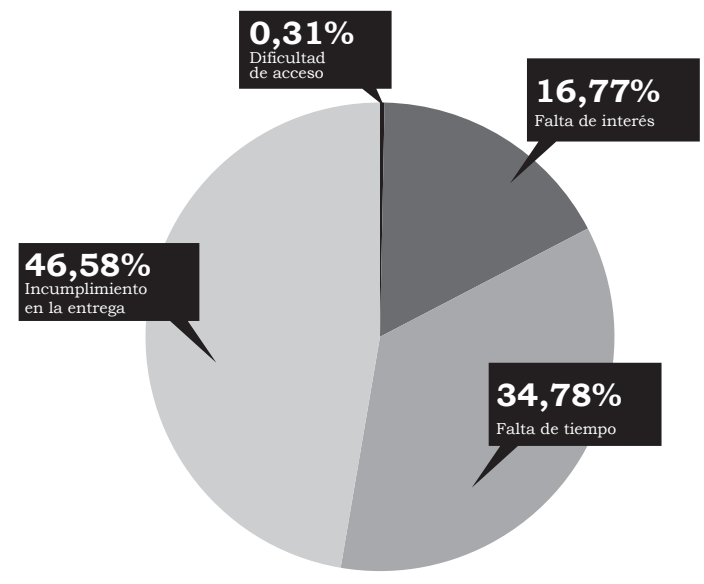

Figura 2. Distribución porcentual de citologías no reclamadas según razón dada por la población en estudio

Fuente: Castro A, Cavieles M, Gutiérrez S. Factores que influyen en la demanda de los resultados de las citologías cervicouterinas en las IPS de Villavicencio durante el año 2008. Villavicencio: Programa de Enfermería, Universidad de los Llanos; 2009.

Por medio de los resultados se ha podido observar que la población del estudio cuenta con condiciones desfavorables para el cuidado de su salud. Esto, junto a la principal causa atribuida por las mujeres para no ir por los resultados, muestra una circunstancia de base complicada por fallas en el proceso. La falta de tiempo aparece como segunda causa, y la falta de interés, como tercera. Esta última solo podría reflejar un conocimiento insuficiente sobre el tema, pues el cuidado de la propia salud no puede carecer de interés para una persona. Diferente a como se esperaba, el miedo al resultado no representa una razón suficiente para tener en cuenta.

\section{Discusión}

Los casos de no reclamación de resultados hallados en el estudio solamente constituyen un $1 \%$ mayor que lo reportado por Profamilia (9\%) (15), pero un $2,7 \%$ menor de lo hallado $(7,3 \%)$ por Barrios García y cols. (16). Este problema fue reseñado por Saslow y cols. (15), quienes lo describen como un problema que interrumpe el proceso de atención y con repercusiones, sobre todo, en las mujeres con resultados anormales que requeririan tratamiento oportuno.

En cuanto al estado civil, la mayoría de las mujeres de la población estudiada tienen pareja estable $(71 \%)$, dato un $21 \%$ mayor que el hallado en el estudio de Restrepo y cols. (17). La diferencia entre solteras en el estudio anterior reporta un $3 \%$ por encima y $2 \%$, en igual sentido, en las separadas. 
Para Barrios García y cols. (17), la no reclamación de los resultados de citología fue del 33,3\% por falta de tiempo, dato muy similar al hallado en el presente trabajo, inferior solo en 1,78\%. Este dato se debe revertir con aplicación de información y comunicación desde las IPS al usuario, a la ver que se debe establecer el costo-beneficio de esta actividad.

Ceballos y cols. (18) reportaron que la mayoría de las mujeres que se realizaron la citología en Soacha reconocieron la importancia del examen en el cuidado de su propia salud. Incluso mencionaron el concepto de autocuidado, en contraste con los resultados obtenidos en este estudio, donde muchas de las mujeres mencionan realizársela como requisito. Se puede notar que este tipo de actitudes podrían ser resultado de la calidad de las estrategias educativas, que deben propender a generar y estimular la cultura del autocuidado, la cual la profesional de enfermería deberá liderar.

La jornada laboral no representó un factor influyente, según los resultados; sin embargo, Lucumí y Gómez (19) hablan de los oficios del hogar como una actividad principal que desarrollan las mujeres y que a menudo no tienen en cuenta. Puede ser que las mujeres de este estudio no consideren sus labores domésticas estrictamente como jornada laboral, y esta termine siendo un factor influyente en la demanda de los resultados.

Cubas Huamán (20) dice: "cuando los resultados se demoran, las mujeres invariablemente pierden interés en el programa y a menudo no regresan", situación que en el presente estudio alcanzó un 46,58\% y que estableció un antecedente negativo para la periodicidad del procedimiento. Una mujer que no regresa al programa de tamizaje es una más con alto riesgo de incrementar la incidencia del cáncer de cuello uterino.

El 34,7\% de la población en estudio manifestó que la falta de tiempo es una de las razones por las cuales no reclaman los resultados de la citología cervicouterina. Este tipo de barreras culturales para la demanda de los resultados de la citología se hacen tangibles cuando las mujeres actúan anteponiendo otras circunstancias (el cuidado de los demás, de la casa y el trabajo), en lugar del cuidado de su salud, en su rol histórico de cuidadoras.

En los estudios, Pinillos-Bolivar (15) observó que un alto porcentaje de la población con bajas condiciones sociodemográficas (estrato 1) no saben para qué sirve la citología que se va a tomar, lo cual es similar en la investigación realizada por Lartey y cols. (21) en el sur de África. Ello podría explicar, en parte, el problema de la no reclamación, dado que siendo de estratos similares, solo cerca del 50\% tenía suficiente conocimiento del mismo tema.

Como dato importante, a mayor edad en la mujer existe mayor probabilidad de asistencia al examen citológico (7). Cercano al presente estudio donde se observa un mayor autocuidado en edades más maduras.

Los resultados encontrados difieren de los de Cubas Huamán (20), quien halló determinantes administrativos como barrera para la realización de la citología, entre ellos el maltrato y falta de información. En el presente estudio estos factores fueron enunciados, pero alcanzaron puntajes porcentuales bajos, esto es, no son determinantes.

Conforme a los resultados obtenidos por Lucía Stella Tamayo y cols. (22), el esposo o la pareja sexual influyen de manera directa e intensa 
en las decisiones que las mujeres toman sobre el cuidado del cuerpo y la salud. En el presente estudio, donde el $71 \%$ tiene pareja, la afirmación anterior se convierte en un dato por indagar, puesto que la no reclamación invisibilizaría en esta población esa influencia del compañero. Es un dato relevante, pero no averiguó qué tanto comparte la usuaria la información de salud y si hay la injerencia a que se refiere la autora precitada, o si el hombre se invisibiliza, esto es, algunos hombres, como parejas, tienden a estar ausentes no solo en los hogares, sino también en el espacio del cuidado de la salud (23), dato que queda pendiente por averiguar.

\section{Conclusión}

La población de nuestro estudio está conformada en su mayoría por mujeres en edad fértil, en unión libre, sin ingresos económicos propios o que devengan menos de un sueldo mínimo; además, tienen estudios incompletos, responsabilidad de cuidar uno o varios hijos, o alguna persona a cargo.

Los resultados demuestran que la plataforma educativa ha llegado eficientemente a la mitad de la población, evidenciada con el grado de conocimiento de las mujeres acerca del examen de la citología.

Las mujeres no muestran la necesidad sentida de acudir a la consulta médica, porque el 91,6\% de las encuestadas asegura acudir al médico solamente cuando se enferman, lo que constituye un factor de riesgo, aunado a la falta de cumplimiento con respecto a la entrega de los resultados por parte de la IPS, que puede prolongar la espera y finalmente la no conclusión del proceso. Son las mismas usuarias las que hablan de este hecho, y le atribuyen directamente la causa de la no demanda de los resultados.

El estudio aporta puntos críticos o débiles del programa en los que se deberá intervenir para fortalecerlo. Se evidencian determinantes administrativos del sistema que desmotivan la reclamación de resultados.

\section{Recomendaciones}

La toma de citología es una responsabilidad del profesional de enfermería, que deberá reforzar su actividad de promoción orientada al compromiso de conocer los resultados, pero a su vez gestionar ante las prestadoras de los servicios de citología, para que haya cumplimiento en la fecha de entrega.

Es vital contar con sistemas de salud ajustados a las normas, aunado a la labor de agentes de salud que no solo operen los procedimientos, sino que estimulen y refuercen los derechos pero también los deberes de los usuarios, son vitales; en gran medida, estos últimos definen la salud y el bienestar de esta población.

Todo Funcionando, el programa de prevención del cáncer cervicouterino, debe mover el engranaje que forja los objetivos institucionales frente a la población, y la entrega del resultado con el tratamiento oportuno. Ello se constituye en el cierre de un proceso que determina la calidad de vida de la usuaria. 
Teniendo en cuenta que en otras instancias está la responsabilidad de mejorar las condicionantes sociales encontradas, sí corresponde al sistema de salud el reforzamiento de la intervención en salud sexual y reproductiva con la mejora de la calidad de los procedimientos involucrados en todo el proceso, considerando fallas fuera del proceso de toma de la citología: calidad del análisis patológico, tratamiento oportuno y seguimiento, que pueden estar contribuyendo al incremento del cáncer cervicouterino, especialmente en esta población de IPS públicas, y que aportan a este grave problema de salud pública.

Teniendo en cuenta que este estudio es realizado solo en IPS públicas y que las usuarias son de ingresos socioeconómicos bajos, cabe señalar que los resultados no se pueden generalizar a usuarias estratos más altos.

\section{Conflicto de interés}

Los autores declaran no tener conflicto de intereses.

\section{Referencias}

1. Merle J. Análisis de la situación del cáncer cervicouterino en América Latina y el Caribe [internet]. Washington, D.C.: Organización Panamericana de la Salud; 2004 [citado 2014 jul 16]. Disponible en: http://bvs.per.paho.org/texcom/cd048376/cacuanal.pdf.

2. Guevara Cuéllar C, Guevara Chaux C, Medina C, Mera Cerón S, Torres Martínez L. Prevalencia de la toma de citología vaginal en estudiantes de una universidad pública de Colombia. 2008 [citado 2014 jul 16];24(1):[about 23-30]. Disponible en: http:// www.scielo.org.co/scielo.php?script $=$ sci_arttext\&pid=S0120$55522008000100004 \& 1 \mathrm{ng}=\mathrm{pt}$.

3. Corredor L, Salazar S, Hincapié D. Cáncer de cuello uterino: factores de riesgo y prevención [internet]. Pereira: Universidad Libre Seccional Pereira; 2009 [citado 2014 jul16]; 6(2):[about 37-45]. Disponible en: file:///C:/Users/upyp/Downloads/6-22-1-PB\%20(2).pdf.

4. Aguilar Pérez JA, Leyva López AG, Angulo Nájera D, Salinas A, Lazcano Ponce EC. Tamizaje en cáncer cervical: conocimiento de la utilidad y uso de citología cervical en México [internet]. 2003 Feb [citado 2014 jul 16];37(1):[about 100-6]. Disponible en: http://www.scielo.br/ scielo.php?script=sci_arttext\&pid=S0034-89102003000100015\&lng.

5. Capurro VI, Rojo JA, Pino T, Vásquez C, Garay J, Venegas M. Programa de Detección y Control de Cáncer de Cuello Uterino en Servicio Salud Araucanía Sur. Rev Chil Obstet Ginecol [internet]. 2002 [citado 2014 jul 16];67(2):[about 114-20]. Disponible en: http://www.scielo.cl/scielo.php?script=sci_arttext\&pid=S07177526200200020000681 lng.

6. Castro Jiménez MA, Londoño Cuellar PA, Vera Cala LM. Asistencia a citología del cuello uterino y sus determinantes en una población 
rural colombiana, 1998-1999. Revista de Salud Pública [internet]. 2006 [citado 2014 jul 16];8(3):[about 248-57]. Disponible en: http:// www.redalyc.org/redalyc-seam/articulo.oa?id=42280310.

7. García Soto G. Cáncer de cuello uterino: experiencia durante un periodo de seis años en un hospital universitario colombiano. Rev Colomb Obstet Ginecol [internet]. 2007 [citado 2014 jul 16];58(1):[about 21-7]. Disponible en: http://www.scielo.org.co/scielo. php?pid=S0034-74342007000100004\&script=sci_arttext.

8. Kasamatsu E, Páez M. Cáncer de cuello uterino y virus del papiloma humano en Paraguay: perspectivas para la prevención primaria. Mem Inst Investig Cienc Salud [internet]. 2006 [citado 2008 abr 8];4(2):[about 58-63]. Disponible en: http://scielo.iics.una.py/scielo. php?pid=S1812-95282006000200011\&script=sci_arttext\&tlng=es.

9. López Guarnizo GA. Estudio del tamizaje citológico para cáncer cervicouterino Laboratorio Departamental de Salud Pública, Caldas, 1998. Hacia la Promoción de la Salud [internet]. 2000 [citado 2008 ago 4];5:[about 1-15]. Disponible en: http://promocionsalud.ucaldas. edu.co/downloads/Revista\%205_12.pdf.

10. Núñez Herrero L, Yanes Calderón M. Citología orgánica: temor o desconocimiento. Rev Cubana Med Gen Integr [internet]. 2007 [citado 2008 abr 8];23:[about 2 p.]. Disponible en: http://scielo.sld.cu/scielo. php?script=sci_arttext\&pid=S0864-21252007000200008\&lng=es.

11. Ojeda G, Ordóñez M, Ochoa LH. Encuesta Nacional de Demografía y Salud 2010: capítulo 7 nupcialidad y exposición al embarazo [internet]. Bogotá: Profamilia-Instituto Nacional de Salud; 2010 [citado 2008 ago 4]; [about 152 p.]. Disponible en: http://www.minsalud.gov. $\mathrm{co} / \mathrm{sites} / \mathrm{rid} /$ Lists/BibliotecaDigital/RIDE/VS/ED/GCFI/Base \%20 de \%20datos \%20ENDS \%202010\%20informe.pdf.

12. Chan MT. La educación y la salud están intimamente unidas [internet]. Washington: Organización Mundial de la Salud; 2010 [citado 2014 mar]; [about 1 p.]. Disponible en: http://www.who.int/dg/speeches/2010/educationandhealth_20100920/es/.

13. Ojeda G, Ordóñez M, Ochoa LH. Encuesta Nacional de Demografía y Salud 2010: detección temprana del cáncer de cuello uterino y de mama [internet]. Bogotá: Profamilia-Instituto Nacional de Salud; 2010 [citado 2008 agosto 4]; [about 441p.]. Disponible en: http:// www.minsalud.gov.co/sites/rid/Lists/BibliotecaDigital/RIDE/VS/ ED/GCFI/Base \%20de \%20datos \%20ENDS \%202010\%20informe. pdf

14. Vasconcelos C, Teixeira M, Pinheiro AKB, Castelo AR, Costa L, Oliveira $\mathrm{R}$. Conhecimento, atitude e prática relacionada ao exame colpocitológico entre usuárias de uma unidade básica de saúde. Rev Latino-Am [internet]. 2011 Feb [citado 2014 jul];19(1): [about 97105 p.]. Disponible en: http://www.scielo.br/scielo.php?script=sci_ arttext\&pid=S0104-11692011000100014\&lng=en.

15. Saslow D et al. American Cancer Society Guideline for Human Papillomavirus (HPV) vaccine use to prevent cervical cancer and 
its precursors. CA Cancer J Clin [internet]. 2007 [citado 2014 abr];57:[about 7-28 p.]. Disponible en: http://onlinelibrary.wiley. com/doi/10.3322/canjclin.57.1.7/pdf.

16. Barrios García L, Benedetti Padrón I, Alvis Estrada L, Salamanca Manjarrez M. Conocimientos, actitudes y prácticas sobre citología cérvico-uterina por mujeres de una población rural en Colombia. Revista Ciencias Biomédicas [internet]. 2013 [citado 2014 abr];4(1):[about 54-9 p.]. Disponible en: http://www.revistacienciasbiomedicas.com/index.php/revciencbiomed/article/view/251.

17. Restrepo Zea J, Mejía Mejía A, Valencia Arredondo M, Tamayo Acevedo L, Salas Zapata W. Accesibilidad a la citología cervical en Medellín, Colombia en 2006. Rev Esp Salud Pública [internet]. 2007 [citado 2014 jul]; 81(6): [about 657-66 p.]. Disponible en: http://scielo.isciii.es/scielo.php?script=sci_arttext\&pid=S113557272007000600009\&lng=es.

18. Wiesner Ceballos C, Vejarano Velandia M, Caicedo Mera JC, Tovar Murillo SL, Cendales Duarte R. La citología de cuello uterino en Soacha, Colombia: representaciones sociales, barreras y motivaciones. Rev Salud Pública [internet]. 2006 [cited 2008 Abril];8(3):[about 185-96 p.]. Disponible en: http://www.scielo.org. co/scielo.php?pid=S0124-00642006000300004\&script=sci_arttext.

19. Lucumí Cuesta DI, Gómez Gutiérrez LF. Accesibilidad a los servicios de salud en la práctica de citología reciente de cuello uterino en una zona urbana de Colombia. Rev Esp Salud Pública [internet]. 2004 [citado 2014 mar];78(3): [about 367-77 p.]. Disponible en: http://scielo.isciii.es/scielo.php?script=sci_arttext\&pid=S113557272004000300006\&lng=es.

20. Cubas Huamán MM. Determinantes administrativos, psicológicos y culturales en la actitud hacia la prueba citológica de cuello uterino de mujeres trujillanas. UCV-Scientia [internet]. 2010 [citado 2014 mar];2(2):[about 34-42 p.]. Disponible en: http://revistas.concytec. gob.pe/pdf/scientia/v2n2/a04v2n2.pdf.

21. Lartey M, Joubert G, Cronje HS. Knowledge, attitudes and practices of rural women in South Africa regarding the Pap smear. Int J Gynaecol Obstet [internet]. 2003 [citado 2014 mar];83(3): [about 315-316 p.].

22. Tamayo LS, Chávez MG, Tamayo Acevedo LE. El cáncer cervicouterino desde la mirada de las mujeres de Antioquia (Colombia) y Colima (México), 2008. Rev Fac Nac Salud Pública [internet]. 2009 [citado 2014 jul 16];27(2):[about 177-86 p.]. Disponible en: http:// www.scielo.org.co/scielo.php?script=sci_arttext\&pid=S0120 386X2009000200009\&lng=en.

23. Díaz Gomez TA. La enfermedad del hombre invisible: imaginarios sociales de masculinidades y feminidades en mujeres residentes en Soacha con diagnóstico de lesiones cervicales asociadas al VPH [disertación]. Facultad de Ciencias Sociales y Humanas: Universidad Externado de Colombia; 2009. 
